\title{
Enhanced Elasticity and Soft Glassy Rheology of a Smectic in a Random Porous Environment
}

\author{
Ranjini Bandyopadhyay ${ }^{1}$, Dennis Liang ${ }^{1}$, Ralph H. Colby ${ }^{2}$, James L. Harden ${ }^{3}$ and Robert L. Leheny ${ }^{1}$ \\ ${ }^{1}$ Department of Physics and Astronomy, \\ Johns Hopkins University, Baltimore, MD 21218, USA \\ ${ }^{2}$ Department of Materials Science and Engineering, \\ Pennsylvania State University, \\ University Park, PA 16802, USA \\ ${ }^{3}$ Department of Chemical and Biomolecular Engineering, \\ Johns Hopkins University, Baltimore, MD 21218, USA
}

(Dated: May 26, 2018)

\begin{abstract}
We report studies of the frequency dependent shear modulus, $G^{*}(\omega)=G^{\prime}(\omega)+i G^{\prime \prime}(\omega)$, of the liquid crystal octylcyanobiphenyl (8CB) confined in a colloidal aerosil gel. With the onset of smectic order, $G^{\prime}$ grows approximately linearly with decreasing temperature, reaching values that exceed by more than three orders of magnitude the values for pure $8 \mathrm{CB}$. The modulus at low temperatures possesses a power-law component, $G^{*}(\omega) \sim \omega^{\alpha}$, with exponent $\alpha$ that approaches zero with increasing gel density. The amplitude of $G^{\prime}$ and its variation with temperature and gel density indicate that the low temperature response is dominated by a dense population of defects in the smectic. In contrast, when the $8 \mathrm{CB}$ is isotropic or nematic, the modulus is controlled by the elastic behavior of the colloidal gel.
\end{abstract}

PACS numbers: 61.30.Pq, 62.25.+g, 61.30.Jf,

Impurities and other forms of quenched disorder can profoundly affect the mechanical properties of materials. For instance, introducing nanometer-scale inclusions into polycrystalline solids is a well-developed approach for enhancing strength by pinning defects whose mobility dictates mechanical compliance [1]. Many complex fluid phases can similarly possess topological defects that influence their viscoelasticity. Important examples are lamellar systems - such as smectic liquid crystals, diblock copolymers, and surfactant mesophases - that are characterized by one-dimensional ordering from the stacking of liquid-like planes. Unlike the case of crystals where impurities can be frozen into the lattice, introducing quenched disorder into such fluids is a technical challenge. In some cases, annealed disorder in the form of colloids suspended in lamellar systems can anchor line defects into a crosslinked network that enhances the elastic response [2, 3].

One systematic approach for introducing quenched disorder to a smectic is to confine the fluid in a random porous medium [4, 5, 6, 7, 8, 9, 10, 11]. Study of such systems has largely concerned the influence of the environment on the phase behavior and smectic ordering. Theory and experiment have concluded that the smectic phase is highly sensitive to the quenched disorder. An interesting example is a smectic confined by an aerosil gel, which is a compliant hydrogen-bonded gel formed from nanometer-scale silica colloids [5, 6, 7, 8, 9, 10]. Consistent with theory 11, 12], x-ray scattering on liquid crystal - aerosil composites has revealed that the gel introduces random field effects that replace the thermodynamically sharp nematic to smectic transition with the growth of short-range smectic correlations $[7,9,10]$. The- ory further predicts that quenched disorder impacts the mechanical behavior of smectics by introducing enhanced anomalous elasticity [11]. We present rheometry studies that characterize how the viscoelastic properties of a smectic are influenced by such disorder. Using weakly bonded aerosil gels enables us to isolate the mechanical response of the smectic. We find that the quenched disorder greatly increases the shear modulus over that of the pure smectic and leads to weak power-law behavior characteristic of soft glassy rheology.

Samples were prepared by forming aerosil gels within the liquid crystal octylcyanobiphenyl (8CB) following established procedures [5]. The 8CB (Frinton Labs) had a quoted purity of $99.4 \%$. Pure $8 \mathrm{CB}$ undergoes an isotropic to nematic transition at $T_{N I}=313.98 \mathrm{~K}$ and a nematic to smectic-A transition in which the molecules form a density wave with period $d_{0}=3.4 \mathrm{~nm}$ at $T_{N A}=306.97$ K [5]. The aerosil (DeGussa Corp., type 300) consists of 7 $\mathrm{nm} \mathrm{SiO}_{2}$ particles that are hydrophilic due to a high density of surface hydroxyl groups. Degassed $8 \mathrm{CB}$ and dried aerosil were mixed with high purity acetone and sonicated for several hours to achieve a uniform dispersion. The mixtures were then heated to $320 \mathrm{~K}$ to evaporate the acetone slowly. During this process the aerosil aggregated via hydrogen bonding. The resulting composites were uniform, thixotropic solids comprised of $8 \mathrm{CB}$ permeated by a colloidal gel. Gels formed in this way have a fractal dimension near 2.1 13] and a broad distribution of void sizes with a mean pore chord $l_{0}$ that varies with aerosil concentration as $l_{0}=6.7 / \rho_{S} \mathrm{~nm}$, where $\rho_{S}$ is the aerosil density in grams $\mathrm{SiO}_{2} / \mathrm{cm}^{3} 8 \mathrm{CB}[8]$. Samples were prepared with $\rho_{S}=0.035$ to $\rho_{S}=0.10$, corresponding to $l_{0} \approx 56 d_{0}$ to $l_{0} \approx 19 d_{0}$ and aerosil volume fractions 
of 0.016 to 0.043 . For $\rho_{S}>0.10$ the composites change from a pasty to a brittle consistency that makes rheometry measurements difficult.

The gel affects both the liquid crystal's nematic and smectic behavior. High porosity gels weakly perturb the orientational ordering, as evidenced by nematic correlation lengths that exceed $l_{0}$ by a factor of 30 or more for $\rho_{S}<0.10$ 14]. The observed random field behavior of the smectic [7] is understood by considering how the randomly positioned gel strands impose steric hinderance on the liquid crystal molecules, effectively forcing the phase of the smectic mass density wave to different values at different points in space. The sensitivity of the smectic ordering to the gel is reflected in the smectic correlation lengths, which are nearly an order of magnitude smaller than the nematic correlation lengths [7].

The frequency-dependent shear modulus, $G^{*}(\omega)=$ $G^{\prime}(\omega)+i G^{\prime \prime}(\omega)$, of the 8CB-aerosil composites was determined using a stress controlled rheometer (Paar Physica MCR300) in a cone and plate geometry equipped with a Peltier device that maintained temperature stability to within $\pm 0.01 \mathrm{~K}$. Temperature gradients across the sample were estimated at less than $0.2 \mathrm{~K}$. Figure 1(a) shows the storage modulus $G^{\prime}(\omega=1 \mathrm{rad} / \mathrm{s})$ as a function of strain amplitude $\gamma_{0}$ for $\rho_{S}=0.10$ at temperatures above and below the smectic transition. When the $8 \mathrm{CB}$ possesses smectic order, the response depends weakly on $\gamma_{0}$ to the smallest strains. However, measurements of $G^{*}(\omega)$ with different $\gamma_{0}$ showed only this slight variation in magnitude; all salient features, particularly the dependence on $\omega$ and temperature, were independent of $\gamma_{0}$ for $\gamma_{0} \leq 0.007$. The results presented below were obtained with $\gamma_{0}=0.003$.

Figures 1(b) and 1(c) show $G^{\prime}(\omega)$ and the loss modulus $G^{\prime \prime}(\omega)$ respectively at several temperatures for $\rho_{S}=0.10$. Above $307 \mathrm{~K}$ the modulus obeys trends of a weak elastic solid, with $G^{\prime}(\omega)$ constant and $G^{\prime}(\omega)>>G^{\prime \prime}(\omega)$, and displays little temperature dependence. In particular, the isotropic to nematic transition near $314 \mathrm{~K}$ has no measurable effect on $G^{*}(\omega)$. We associate this high temperature mechanical response with that of the aerosil gel in a low viscosity liquid crystal solvent. Measurements of aerosil gels in the simple organic liquid dibutyl phthalate give quantitatively similar $G^{*}(\omega)$, supporting this interpretation. The storage modulus $G_{g}$ above the smectic transition temperature varies with aerosil density roughly as $G_{g} \sim \rho_{S}^{4.8 \pm 0.8}$, consistent with behavior expected for a colloidal gel with fractal dimension near 2.1 [15]. With the onset of smectic ordering in the $8 \mathrm{CB}$ at low temperatures, $G^{\prime}(\omega)$ increases dramatically and obtains a clear frequency dependence. We attribute the growth in $G^{*}(\omega)$ to contributions from smectic defects. The solid lines in Fig. 1(b) are the results of fits to a power-law contribution plus a frequency-independent background

$$
G^{\prime}(\omega)=G_{g}+G_{s}+\beta_{s} \omega^{\alpha} .
$$

where $G_{g}$ is the contribution from the gel, assumed independent of $\omega$ and temperature, and $G_{s}(T)$ is the

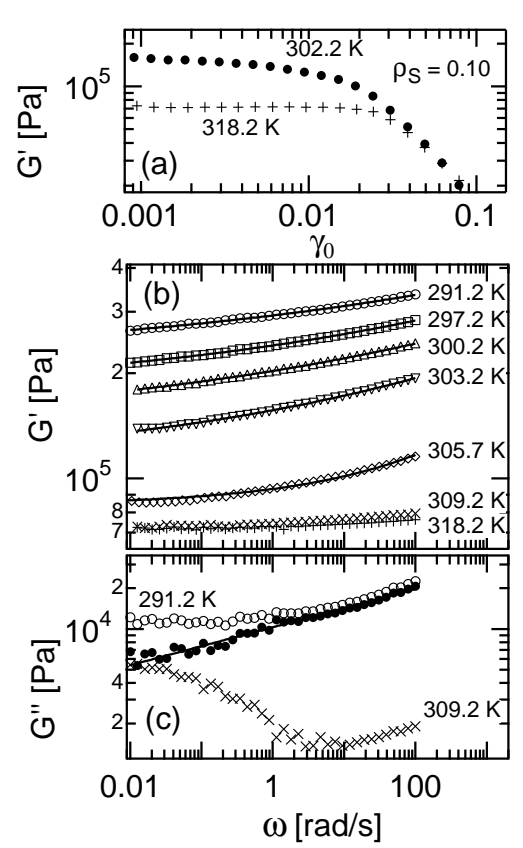

FIG. 1: (a) Storage modulus $G^{\prime}$ of $8 \mathrm{CB}$ confined in an aerosil gel with $\rho_{S}=0.10 \mathrm{~g} / \mathrm{cm}^{3}$ at $\omega=1 \mathrm{rad} / \mathrm{s}$ as a function of strain amplitude $\gamma_{0}$ below (solid circles) and above $(+)$ the smectic transition temperature, $T_{N A}$. (b) $G^{\prime}(\omega)$ at several temperatures for $\gamma_{0}=0.003$. Lines are results of fits to Eq. (1). (c) Loss modulus $G^{\prime \prime}(\omega)$ below (open circles) and above (Xs) $T_{N A}$. The solid circles display the difference between the two spectra, and the line is the result of a power-law fit.

frequency-independent contribution from the smectic phase. This form describes accurately the storage modulus at all densities of aerosil and at all temperatures.

The inset to Fig. 2 displays the amplitudes for the power-law and frequency-independent contributions, $\beta_{s}$ and $G_{s}$, as a function of temperature for $\rho_{S}=0.10$. These parameters show qualitatively the same temperature dependence for all $\rho_{S}$ except that the ratio $\beta_{s} / G_{s}$ increases with increasing $\rho_{S}$. The exponent $\alpha$ characterizing the power-law dependence displays no systematic temperature dependence below the pseudocritical point marking the onset of static short-range smectic order [7, 8] but does vary systematically with $\rho_{S}$. Figure 2 displays the value of $\alpha$ averaged over temperature as a function of $\rho_{S}$ wherein $\alpha$ decreases from $\alpha \approx 0.25$ at $\rho_{S}=0.035$ to $\alpha \approx 0.1$ at $\rho_{S}=0.10$. Such weak power-law behavior is characteristic of a variety of complex fluids including foams, emulsions, particulate suspensions and slurries. In a recent model Sollich and coworkers 16] provide a unifying theoretical framework for this "soft glassy rheology", arguing that the response is a general consequence of structural disorder and metastability. In this model $\alpha$ serves as an effective noise temperature, with systems entering a glass transition as $\alpha \rightarrow 0$. (The exponent $x$ in Ref. 16] is $x=\alpha+1$.) Thus, the variation of $\alpha$ with $\rho_{S}$ points to increasingly glassy dynamics in the smectic 


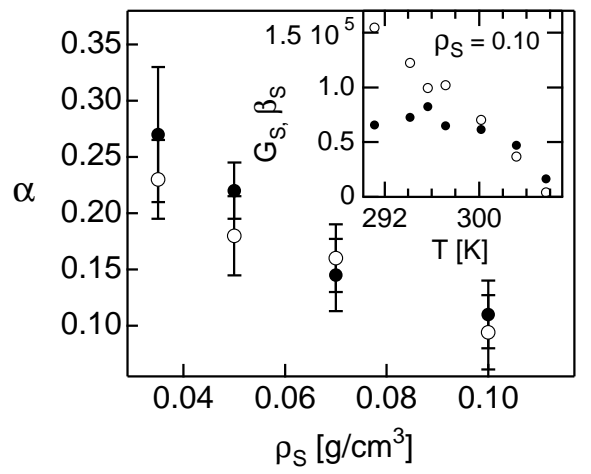

FIG. 2: The exponent $\alpha$ characterizing the power-law contribution to $G^{\prime}(\omega)$ (solid circles) and $G^{\prime \prime}(\omega)$ (open circles) for smectic $8 \mathrm{CB}$ confined by an aerosil gel as a function of gel density. The inset displays results for $G_{s}$ (open circles) and $\beta_{s}$ (filled circles) corresponding to the amplitudes of the frequency-independent and power-law contributions to $G^{\prime}(\omega)$, respectively, for $8 \mathrm{CB}$ in a gel with $\rho_{S}=0.10 \mathrm{~g} / \mathrm{cm}^{3}$ at temperatures below the onset of smectic order in the $8 \mathrm{CB}$.

with increased quenched disorder. This notion of glassy behavior further connects the rheology with the slow dynamics observed with dynamic light scattering for $8 \mathrm{CB}$ in aerosil [17]. However, we note the simultaneous growth of the frequency-independent component $G_{s}$ implies some portion of the $8 \mathrm{CB}$ is frozen for all $\rho_{S}$.

As expected for soft glassy rheology [16], the weak power-law behavior also appears in the loss modulus. Figure 1(c) displays $G^{\prime \prime}(\omega)$ at low temperature in the smectic phase of $8 \mathrm{CB}$ and at high temperature in the nematic phase, where contributions from the gel presumably dominate. With the high temperature modulus subtracted from the low temperature spectrum, the remaining smectic contribution, shown by the solid symbols in Fig. 1(c), displays power-law behavior. As shown in Fig. 2, fits to such results, $G^{\prime \prime}(\omega) \sim G^{\prime}(\omega) \sim \omega^{\alpha}$, provide values for $\alpha$ that agree closely with those obtained from $G^{\prime}(\omega)$.

The amplitude and frequency dependence of $G^{\prime}(\omega)$ for smectic 8CB in aerosil differ from those of other unaligned lamellar complex fluids. A wide range of systems including lyotropic and thermotropic smectics (without quenched disorder), surfactant-based phases, and diblock copolymers exhibit a frequency response with two contributions: $G^{\prime}(\omega)=G_{0}+\beta_{d} \omega^{1 / 2}$. The plateau modulus $G_{0}$ arises from the elasticity of static defects while the frequency-dependent term results from the bulk response of the material. Specifically, $\beta_{d} \omega^{1 / 2}$ with $\beta_{d}=$ $(\pi / 24 \sqrt{2}) \sqrt{(B \eta)}$, where $B$ is the compression modulus and $\eta$ is an effective viscosity, describes the response of lamellar regions with the layer normal oriented so that strain necessitates layer compression [18]. While the relative strength of these terms varies with material parameters and defect structures, most studies on unaligned lamellar systems capture this characteristic response in

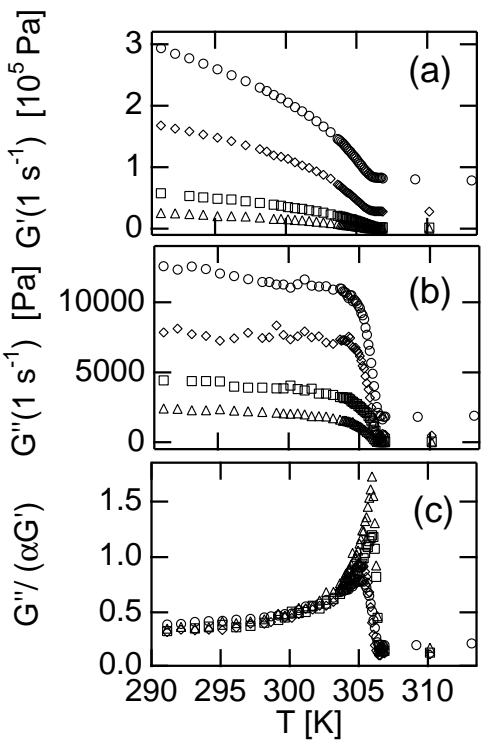

FIG. 3: (a) Storage modulus $G^{\prime}(T)$ and (b) loss modulus $G^{\prime \prime}(T)$ at $\omega=1 \mathrm{rad} / \mathrm{s}$ for $8 \mathrm{CB}$ in an aerosil gel with $\rho_{S}=$ $0.035 \mathrm{~g} / \mathrm{cm}^{3}$ (triangles), $0.05 \mathrm{~g} / \mathrm{cm}^{3}$ (squares), $0.07 \mathrm{~g} / \mathrm{cm}^{3}$ (diamonds), and $0.10 \mathrm{~g} / \mathrm{cm}^{3}$ (circles). (c) The ratio $G^{\prime \prime} / G^{\prime}$ scaled by the exponent $\alpha$ for the four densities.

their experimental frequency range. In particular, experiments on pure unaligned $8 \mathrm{CB}$ display a plateau modulus $G_{0} \approx 100 \mathrm{~Pa}$ and a $\omega^{1 / 2}$ contribution with $\beta_{d} \approx 30$ $\mathrm{Pa} \cdot \mathrm{s}^{1 / 2}[19,20$, consistent with the room temperature values $B \approx 2 \cdot 10^{6} \mathrm{~Pa}$ and $\eta \approx 0.1 \mathrm{~Pa} \cdot \mathrm{s}$. The response we observe for $8 \mathrm{CB}$ in aerosil is more than three orders of magnitude larger than $G^{\prime}(\omega)$ of pure $8 \mathrm{CB}$. The greatly enhanced modulus in the presence of disorder implies that contributions from the bulk fluid are negligible. Instead, we infer that the shear response derives from a dense population of defects that form in the smectic with the destruction of smectic correlations by the disorder. Constraints imposed on defect motion by the confining gel provide rigidity that dominates the glassy response to shear.

Evidence for soft glassy rheology and the approach to a glass transition with increasing $\rho_{S}$ comes not only from the weak power-law behavior of the modulus but also from the ratio $G^{\prime \prime} / G^{\prime}$. Figures 3(a) and 3(b) show the temperature dependence of $G^{\prime}$ and $G^{\prime \prime}$, respectively, at $\omega=1 \mathrm{rad} / \mathrm{s}$ for varying $\rho_{S}$. Within the soft glassy rheology model $G^{\prime \prime} / G^{\prime} \sim \alpha[16]$. Figure 3(c) shows $G^{\prime \prime} / G^{\prime}$ scaled by $\alpha$. At temperatures below the pseudocritical region, the ratios collapse onto a single scaling function. This scaling demonstrates how the single parameter $\alpha$ accounts for the variations in mechanical response with changing $\rho_{S}$. Thus, a direct correspondence exists between the strength of random fields in the system, as parameterized by $\rho_{S}[7,[8]$, and the exponent $\alpha$ that is central to soft glassy rheology.

The variation of $G^{\prime}$ with $\rho_{S}$ indicates that smectic line 


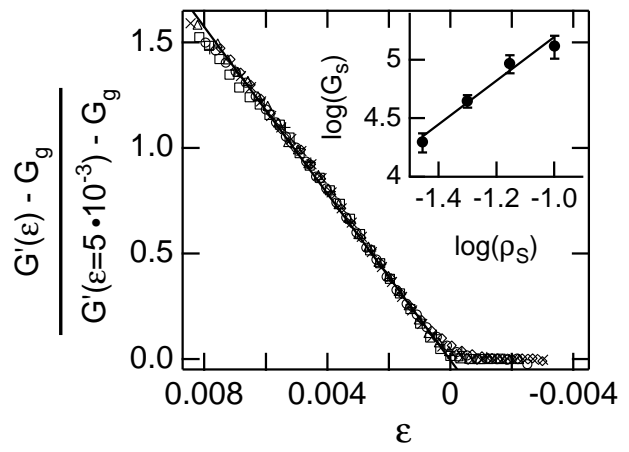

FIG. 4: Smectic contribution to $G^{\prime}$ near the pseudotransition temperature that marks the onset of smectic order normalized by its value at the reduced temperature $\epsilon=5 \cdot 10^{-3}$ for $8 \mathrm{CB}$ in an aerosil gel. Included are $G^{\prime}(\omega=1)$ for $\rho_{S}=0.035 \mathrm{~g} / \mathrm{cm}^{3}$ (triangles), $0.05 \mathrm{~g} / \mathrm{cm}^{3}$ (squares), $0.07 \mathrm{~g} / \mathrm{cm}^{3}$ (diamonds), and $0.10 \mathrm{~g} / \mathrm{cm}^{3}$ (circles) as well as $G^{\prime}(\omega=0.1)$ (crosses) and $G^{\prime}(\omega=40)$ (X's) for $0.07 \mathrm{~g} / \mathrm{cm}^{3}$. The solid line represents a linear dependence on $\epsilon$. The inset displays the values of $G_{s}$ at $292.6 \mathrm{~K}$ with the solid line showing the fit result $G_{s} \sim \rho_{S}^{1.9 \pm 0.2}$.

defects dominate this soft glassy rheology. In analogy with rubber elasticity, the elastic response of a static defect network should vary as $G_{s} \approx \tau / d^{2}$ where $\tau$ is the defect line tension and $d$ is a typical spacing between defects 22. A plausible scale for $d$ is set by the porosity of the gel. Assuming $d \approx \xi \sim l_{0}$, where $\xi$ is the smectic correlation length and $l_{0}$ is the gel's mean pore chord [5, 7], we expect $G_{s} \sim l_{0}^{-2} \sim \rho_{S}^{2}$. The inset to Fig. 4 displays $G_{s}$ at $292.6 \mathrm{~K}$, well below the nematic to smectic transition, as a function of $\rho_{S}$. The solid line in the inset represents the result of the best fit to a power law, $G_{s} \sim \rho_{S}^{1.9 \pm 0.2}$, consistent with this expectation.

The temperature dependence of $G^{\prime}$ similarly indicates defects dominate the mechanical response. Figure 4 shows the smectic contribution to $G^{\prime}$ as a function of reduced temperature, $\epsilon \equiv\left(T_{N A}-T\right) / T_{N A}$, normalized by its value at $\epsilon=5 \cdot 10^{-3}$ 21]. Included in the plot are data from Fig. 3(a) covering $G^{\prime}(\omega=1, \epsilon)$ for four aerosil densities as well as $G^{\prime}(\omega=0.1, \epsilon)$ and $G^{\prime}(\omega=40, \epsilon)$ for $\rho_{S}=0.07$. The data collapse onto the solid line which displays the relation $\left(G^{\prime}(\epsilon)-G_{g}\right) \sim \epsilon^{z}$ with $z=1$. This scaling demonstrates that the smectic contribution to the mechanical response is associated with critical phenomena of the nematic to smectic transition. As mentioned above, analogy with rubber elasticity predicts $G^{\prime} \sim \tau$, where $\tau$ is the defect line tension. For screw dislocations, which are prominent in smectics [22], $\tau=B b^{4} / 128 \pi^{3} r_{c}^{2}$, where $b=m d_{0}$ is the Burger's vector of integer strength $m$ and $r_{c}$ is the defect core radius 23]. For $8 \mathrm{CB}$ near $T_{N A}, B \sim \epsilon^{0.4}\left[24\right.$ and $r_{c}^{-2} \sim \psi^{2} \sim \epsilon^{0.5}$, where $\psi$ is the smectic order parameter 7 ]. Thus, $\tau \sim \epsilon^{0.9}$ in close agreement with $z \approx 1$ we observe for $G^{\prime}$, suggesting that screw dislocations play a predominant role in dictating the shear response. A more quantitative assessment of the prominence of screw dislocations depends on the typical Burger's vector strength. For a moderate value, $m=4$, along with the low temperature values $B \approx 10^{7}$ $\mathrm{Pa}$ [24], $r_{c} \approx 1 \mathrm{~nm}$, and $G_{s}$ from the inset of Fig. 4, the relation $G_{s} \approx \tau / d^{2}$ leads to a plausible defect spacing $d \approx 0.5 l_{0}$, independent of aerosil density.

Regardless of the precise connection between the temperature dependence of $G^{\prime}$ and quenched disorder, the observed enhanced elasticity and soft glassy rheology of $8 \mathrm{CB}$ confined in aerosil provides a new perspective on effects of quenched disorder in complex fluids. Unlike for many soft glassy systems, the defects that drive this rheology provide a clear physical origin for the disorder and metastability in the smectic. Furthermore, the quenched disorder that the gel imposes on the smectic has been convincingly characterized in terms of random fields [, 11]. Thus, this mechanical behavior connects a classic model of quenched disorder, from random fields, with a new paradigm of glassy behavior in complex fluids.

We thank B. Erwin and R. Patil for assistance and Z. Tešanović for helpful discussions. Funding was provided by the NSF (DMR-0134377).
[1] F. A. McClintock and A. S. Argon, Mechanical Properties of Materials (Addison-Wesley, New York, 1966).

[2] L. Ramos et al., Phys. Rev. E 66, 031711 (2002).

[3] G. Basappa et al., Eur. Phys. J. B 12, 269 (1999).

[4] T. Bellini et al., Science 294, 1074 (2001).

[5] G. S. Iannacchione et al., Phys. Rev. E 58, 5966 (1998).

[6] T. Jin and D. Finotello, Phys. Rev. Lett. 86, 818 (2001).

[7] R. L. Leheny et al., Phys. Rev E 67, 011708 (2003).

[8] G. S. Iannacchione et al., Phys. Rev E 67, 011709 (2003).

[9] P. S. Clegg et al., Phys. Rev E 67, 021703 (2003).

[10] D. Liang, M. A. Borthwick, and R. L. Leheny, J. Phys.: Condens. Matter 16, S1989 (2004).

[11] L. Radzihovsky and J. Toner, Phys. Rev. B 60, 206 (1999).

[12] P. D. Olmsted and E. M Terentjev, Phys. Rev. E 53, 2444 (1996).
[13] G. S. Iannacchione, (unpublished).

[14] T. Bellini et al., Phys. Rev. E 57, 2996 (1998).

[15] R. Buscall et al., J. Chem. Soc., Faraday Trans. 184 , 4249 (1988).

[16] P. Sollich et al., Phys. Rev. Lett. 78, 2020 (1997).

[17] A. Mertelj et al., Mol. Cryst. Liq. Cryst. 331, 81 (1999).

[18] K. Kawasaki and A. Onuki, Phys. Rev. A 42, 3664 (1990).

[19] R. G. Larson et al., Rheol. Acta 32, 245 (1993).

[20] R. H. Colby et al., Rheol. Acta 36, 498 (1997).

[21] The values for $T_{N A}$ were $306.16 \mathrm{~K}$ for $\rho_{S}=0.035,306.35$ $\mathrm{K}$ for $\rho_{S}=0.05,305.93 \mathrm{~K}$ for $\rho_{S}=0.07$, and $306.18 \mathrm{~K}$ for $\rho_{S}=0.10$, consistent with previous studies $[5,7]$

[22] J. A. N. Zasadzinski, J. Phys. France 51, 747 (1990).

[23] M. Kléman, Rep. Prog. Phys. 52, 555 (1989).

[24] M. Benzekri et al., Phys. Rev. B 41, 9032 (1990). 